\title{
A CONVERGENCE ANALYSIS FOR NEWTON-LIKE METHODS IN BANACH SPACE UNDER WEAK HYPOTHESES AND APPLICATIONS
}

\author{
IOANNIS K. ARGYROS
}

\begin{abstract}
In this study we use Newton-like methods to approximate solutions of nonlinear equations in a Banach space setting. Most convergence results for Newton-like methods involve some type of a Lipschitz continuity condition on the frechet-derivative of the operator involved. However there are many interesting real life problems already in the literature where the operator can only satisfy a Holder continuity condition. That is why here we chose the Frechet-derivative of the operator involved to be only Holder continuous, which allows us to consider a wider range of problems than before. Special choices of our parameters reduce our results to earlier ones. An error analysis is also provided for our method. At the end of our study, we provide applications to show that our results apply where earlier results do not. In paricular we solve a two point boundary value problem appearing in physics in connection with the problem of bending of beams.
\end{abstract}

\section{Introduction}

In this study we are concerned with the problem of approximating a locally unique solution $x^{*}$ of the equation

$$
F(x)=0,
$$

where $F$ is a Frechet-differentiable nonlinear operator defined on some open convex subset $D$ of a Banach space $E_{1}$ with values in a Banach space $E_{2}$.

Newton-like methods of the form

$$
x_{n+1}=x_{n}-A\left(x_{n}\right)^{-1} F\left(x_{n}\right) \quad(n \geq 0)
$$

for some initinal guess $x_{0} \in D$ have been used extensively to approximate a solution $x^{*}$ of equation. (1) (see, e.g. [1]-[9]). Here the operator $A$ which maps $E_{1}$ into $L\left(E_{1}, E_{2}\right)$, the set of bounded linear operators from $E_{1}$ into $E_{2}$, is an approximation to the Frechetderivative $F^{\prime}(\cdot)$ of the operator $F$. Note that the usual choices for the operator $A$ are given by $A(x)=F^{\prime}(x)$ for all $x \in D$ (Newton's method) or $A\left(x_{n}\right)=\delta F\left(x_{n}, x_{n-1}\right) n \geq 0$ (Secant method) [1]-[5]. Other choices are also possible [1], [8], [9], [10].

Received September 17, 1996; revised February 241999.

1991 Mathematics Subject Classification. 47H17, 65J15, 49M15.

Key words and phrases. Newton-like methods, Banach, space, nondiscrete induction. 
The main hypotheses in most works summarizes to Lipschitz continuity conditions:

$$
\| A\left(x_{0}\right)^{-1}\left(A(x)-A\left(x_{0}\right) \mid \leq k_{1}\left\|x-x_{0}\right\|+k_{0},\right.
$$

and

$$
\left\|A\left(x_{0}\right)^{-1}\left(F^{\prime}(x+t(y-x))-A(x)\right)\right\| \leq k_{2} t\|x-y\|+k_{3}\left\|x-x_{0}\right\|+k_{4}
$$

for all $x, y \in U\left(x_{0}, R\right)=\left\{x \in E_{1}\left\|x-x_{0}\right\| \leq R\right\} \subseteq D, t \in[0,1]$, some fixed $x_{0}, R, k_{0}, k_{1}$, $k_{2}, k_{3}, k_{4}$ with $x_{0} \in D$ such that $A\left(x_{0}\right)$ is invertible, and $R, k_{0}, k_{1}, k_{2}, k_{3}, k_{4} \geq 0$.

However there are many interesting problems in the literature (see e.g. [1], [7] and the examples at the end of this study), where conditions (3) and (4) are not satisfied. Here, we only assume that Holder continuity conditions are satisfied:

$$
\| A\left(x_{0}\right)^{-1}\left(A(x)-A\left(x_{0}\right) \mid \leq c_{1}\left\|x-x_{0}\right\|^{p}+c_{0},\right.
$$

and

$$
\left\|A\left(x_{0}\right)^{-1}\left(F^{\prime}(x+t(y-x))-A(x)\right)\right\| \leq c_{2}(t\|x-y\|)^{p}+c_{3}\left\|x-x_{0}\right\|^{p}+c_{4},
$$

for all $x, y \in U\left(x_{0}, R\right), t \in[0,1]$, some fixed $x_{0}, R, c_{0}, c_{1}, c_{2}, c_{3}, c_{4}$ with $x_{0} \in D$ such that $A\left(x_{0}\right)$ is invertible, $R \geq 0$ such that $U(x, 0, R) \subseteq D, p \in(0,1]$, and $c_{0}, c_{1}, c_{2}, c_{3}, c_{4} \geq 0$.

For $p=1, c_{0}=k_{0}$ and $c_{1}=k_{1}(5)$ reduces to (3), whereas for $p=1, c_{2}=k_{2}, c_{3}=k_{3}$ and $c_{4}=k_{4}(6)$ reduces to (4). These conditions allow us to consider a wider range of problems than before [1] - [9].

We approximate a locally unique solution $x^{*}$ of equation (1), as well as provide precise error estimates on the distances

$$
\left\|x_{n+1}-x_{n}\right\| \text { and }\left\|x_{n}-x^{*}\right\| \quad(n \geq 0) .
$$

Moreover our convergence results reduce to earlier ones [1] -[9] for the above stated choices of operator $A$ and constants $p, c_{0}, c_{1}, c_{2}, c_{3}$ and $c_{4}$.

At the enid of this study, we provide two examples, one from a real scalar equation, and one involving the solution of a two point boundary value problem appearing in physics (bending of beams problems [4], [5]) where, we show. that earlier results do not apply, whereas ours can.

\section{Convergence Analysis}

We can now formulate the following semi-local result on the convergence of the Newton-like method generated by approximation (2).

Theorem. Assume:

(i) nonlinear operator $F$ is defined on an open convex subset $D$ of a Banach space $E_{1}$ with values on a Banach space $E_{2}$;

(ii) $F$ is Frechet-differentiable on $D$; 
(iii) operator $A(x) \in L\left(D, E_{2}\right)(x \in D)$ and there exists a fixed $x_{0} \in D$ such that the linear operator $A\left(x_{0}\right)$ is blundedly invertible on $D$;

(iv) conditions (5) and (6) are satisfied for some fixed $p \in(0,1], R \geq 0$ and all $x, y \in$ $U\left(x_{0}, R\right)$;

(v) ball $U\left(x_{0}, R\right) \subseteq D$;

(vi) there exists a smallest nonnegative number d satisfying the inequatlities

$$
f(d) \leq 0 \text { and } d \leq R
$$

where

$$
\begin{gathered}
f(r)=-r+\eta+\frac{\alpha(r)^{-\frac{1}{p}}\left(\alpha(r)^{\frac{1}{p}} \eta\right)^{1+p}}{1-\left(\alpha^{\frac{1}{p}}(r) \eta\right)^{p}}+\frac{\beta(r)}{1-\beta(r)} \eta, \\
\eta \geq\left\|-A\left(x_{0}\right)^{-1} F\left(x_{0}\right)\right\|, \alpha(r)=\frac{c_{2}}{(1+p)\left[1-\left(c_{1} r^{p}+c_{0}\right)\right]}, \text { and } \beta(r)=\frac{c_{3} r^{p}+c_{4}}{1-\left(c_{1} r^{p}+c_{0}\right)} \text { on }[0, \mathrm{R}] .
\end{gathered}
$$

(vii) Moreover the parameters $\eta, d, R$ satisfy:

$$
\frac{c_{2}}{1+p}(d+R)^{p}+c_{3} R^{p}+c_{4}+c_{1} R^{p}+c_{0}<1
$$

where $a=\alpha(d)$ and $q=\beta(d)$.

Then, Newton-like iteration $\left\{x_{n}\right\} \cdot(n \geq 0)$ generated by approximation $(2)$ is well defined, remains in $U\left(x_{0}, d\right)$ for all $n \geq 0$ and converges to a solution $x^{*}$ of equation (1), which is unique in $U\left(x_{0}, R\right)$. Moreover, the following estimates are true:

$$
\begin{aligned}
\left\|x_{n+1}-x_{n}\right\| \leq & \frac{1}{1-\left[c_{1}\left\|x_{n}-x_{0}\right\|^{p}+c_{0}\right]}\left[\frac{c_{2}}{1+p}\left\|x_{n}-x_{n-1}\right\|^{1+p}\right. \\
& \left.+\left(c_{3}\left\|x_{n-1}-x_{0}\right\|^{p}+c_{4}\right)\left\|x_{n}-x_{n-1}\right\|\right] \\
\leq & a^{-\frac{1}{p}} b^{(1+p)^{n}}+q \frac{1-q^{n}}{1-q} \eta \quad(n \geq 1), \\
\left\|x_{n}-x_{0}\right\| \leq & a^{-\frac{1}{p}}\left[b^{1+p}+\cdots+\left(b^{1+p}\right)^{n-1}\right]+q \frac{1-q^{n-1}}{1-q} \eta+\eta \leq d \quad(n \geq 2)
\end{aligned}
$$

and

$$
\left\|x_{n}-x^{*}\right\| \leq \frac{a^{-\frac{1}{p}} b^{(1+p) n}}{1-b^{p}}+\frac{q^{\eta}}{1-q} \eta \quad(n \geq 1)
$$

where

$$
b=a^{\frac{1}{p}} \eta
$$

Proof. The point $x_{1} \in U\left(x_{0}, d\right)$, since $\eta \leq d$. We assume that $x_{k} \in U\left(x_{0}, d\right)$, for $k=0,1,2, \ldots, n$. Using condition (5), we obtain

$$
\left\|A\left(x_{0}\right)^{-1}\left(A\left(x_{k}\right)-A\left(x_{0}\right)\right)\right\| \leq c_{1}\left\|x_{k}-x_{0}\right\|^{p}+c_{0} \leq c_{1} d^{p}+c_{0}<1,
$$


by hypotheses (7) and (9). It now follows from relation (13) and the Banach lemma on invertible operators [5] that the linear operator $A\left(x_{k}\right)$ is invertible and

$$
\left\|A\left(x_{k}\right)^{-1} A\left(x_{0}\right)\right\| \leq \frac{1}{1-\left[c_{1}\left\|x_{k}-x_{0}\right\|^{p}+c_{0}\right]} \leq \frac{1}{1-\left[c_{1} d^{p}+c_{0}\right]} .
$$

By approximation (2), we get

$$
F\left(x_{k}\right)=\int_{0}^{1}\left[F^{\prime}\left(x_{k-1}+t\left(x_{k}-x_{k-1}\right)\right)-A\left(x_{k-1}\right)\right]\left(x_{k}-x_{k-1}\right) d t
$$

and by (6)

$$
\begin{aligned}
\left\|A\left(x_{0}\right)^{-1} F\left(x_{k}\right)\right\| & \leq\left\|\int_{0}^{1} A\left(x_{0}\right)^{-1}\left[F^{\prime}\left(x_{k-1}+t\left(x_{k}-x_{k-1}\right)\right)-A\left(x_{k-1}\right)\right] d t\left(x_{k}-x_{k-1}\right)\right\| \\
& \leq\left[\frac{c_{2}}{1+p}\left\|x_{k}-x_{k-1}\right\|^{p}+c_{3}\left\|x_{k-1}-x_{0}\right\|^{p}+c_{4}\right]\left\|x_{k}-x_{k-1}\right\| .
\end{aligned}
$$

Hence, by relation (2), (14), (15) and the induction hypotheses, we obtain

$$
\begin{aligned}
\left\|k_{k+1}-x_{k}\right\| \leq & \left\|A\left(x_{k}\right)^{-1} A\left(x_{0}\right)\right\| \cdot\left\|A\left(x_{0}\right)^{-1} F\left(x_{k}\right)\right\| \\
\leq & \frac{1}{\left[1-\left(c_{1}\left\|x_{k}-x_{0}\right\|^{p}+c_{0}\right)\right]}\left[\frac{c_{2}}{1+p}\left\|x_{k}-x_{k-1}\right\|^{1+p}\right. \\
& \left.\quad+\left(c_{3}\left\|x_{k-1}-x_{0}\right\|^{p}+c_{4}\right)\left\|x_{k}-x_{k-1}\right\|\right] \leq \cdots \leq \\
\leq & a^{(1+p)^{0}+(1+p)+\cdots+(1+p)^{k-1}} \eta^{(1+p)^{k}}+q^{k} \eta \\
\leq & a^{\frac{(1+p)^{k}-1}{1+p-1}} \eta^{(1+p)^{k}}+q^{k} \eta=a^{-\frac{1}{p} b^{(1+p)^{k}}+q^{k} \eta,}
\end{aligned}
$$

which shows (10) for all $n \geq 1$.

Moreover by relation (16) and the triangle inequality, we obtain in turn

$$
\begin{aligned}
\left\|x_{k+1}-x_{0}\right\| & \leq\left\|x_{k+1}-x_{k}\right\|+\left\|x_{k}-x_{k-1}\right\|+\cdots+\left\|x_{1}-x_{0}\right\| \\
& \leq a^{-\frac{1}{p}}\left[b^{1+p}+b^{(1+p)^{2}}+\cdots+b^{(1+p)^{k}}\right]+\left(q+q^{2}+\cdots+q^{k}\right) \eta+\left\|x_{1}-x_{0}\right\| \\
& \leq a^{-\frac{1}{p}}\left[b^{1+p}+b^{1+2 p}+\cdots+b^{1+k p}\right]+q \frac{1 \cdot-q^{k}}{1-q} \eta+\left\|x_{1}-x_{0}\right\| \\
& =a^{-\frac{1}{p}} b^{1+p}\left(\frac{1-b^{(k+1) p}}{1-b^{p}}\right)+\frac{q}{1-q} \eta+\eta \\
& \leq \frac{a^{-\frac{1}{p}} b^{1+p}}{1-b^{p}}+\frac{q}{1-q} \eta+\eta \leq d, \text { by hypothesis }(7) .
\end{aligned}
$$

That is, we deduce that $x_{k+1} \in U\left(x_{0}, d\right)$.

For $m \in N$, we have in turn

$$
\begin{aligned}
\left\|x_{n+m}-x_{n}\right\| & \leq\left\|x_{n+m}-x_{n+m-1}\right\|+\left\|x_{n+m-1}-x_{n+m-2}\right\|+\cdots+\left\|x_{n+1}-x_{n}\right\| \\
& \leq a^{-\frac{1}{p}} b^{n(1+p)} \frac{1-b^{(m+1) p}}{1-b^{p}}+q^{n} \frac{1-q^{m+1}}{1-q} \eta .
\end{aligned}
$$


Hence iteration $\left\{x_{n}\right\}$, $(n \geq 0)$ forms a Cauchy sequence and remains in $U\left(x_{0}, d\right)$. Thus it tends to some $x^{*} \in U\left(x_{0}, d\right)$. Letting $m$ tend to infinity in relation (17), we obtain relation (12). Using relation (12) for $m=0$ we get $x^{*} \in U\left(x_{0}, d\right)$, and by letting $k$ tend to infinity in relation (15), we obtain $F\left(x^{*}\right)=0$, which shows that $x^{*}$ is a solution of equation (1). To show uniqueness, let us assume that there exists another solution $y^{*}$ of equation (1) in $U\left(x_{0}, R\right)$. Then from the estimate

$$
\begin{aligned}
& \| \int_{0}^{1} A\left(x_{0}\right)^{-1}\left(\int_{0}^{1}\left[F^{\prime}\left(y^{*}+t\left(x^{*}-y^{*}\right)-A\left(y^{*}\right)+A\left(y^{*}\right)-A\left(x_{0}\right)\right]\right) d t \|\right. \\
\leq & \frac{c_{2}}{1+p}\left\|x^{*}-y^{*}\right\|^{p}+c_{3}\left\|y^{*}-x_{0}\right\|^{p}+c_{4}+c_{1}\left\|x_{0}-y^{*}\right\|^{p} \\
\leq & \frac{c_{2}}{1+p}\left(\left\|x^{*}-x_{0}\right\|+\left\|x_{0}-y^{*}\right\|\right)^{p}+c_{3} R^{p}+c_{4}+c_{1}\left\|x_{0}-y^{*}\right\|^{p} \\
\leq & \frac{c_{2}}{1+p}(d+R)^{p}+c_{3} R^{p}+c_{4}+c_{1} R^{p}<1,
\end{aligned}
$$

by hypothesis (9). It now follows that the linear operator $\int_{0}^{1} F^{\prime}\left(y^{*}+t\left(x^{*}-y^{*}\right)\right) d t$ is invertible, and from the approximation

$$
F\left(x^{*}\right)-F\left(y^{*}\right)=\int_{0}^{1} F^{\prime}\left(y^{*}+t\left(x^{*}-y^{*}\right)\right) d t\left(x^{*}-y^{*}\right),
$$

it follows that $x^{*}=y^{*}$, which completes the proof of the theorem.

Remarks. The results obtained in the Theorem do not compare favorably with the ones by Kantorowitsch [5] for $A(x)=F^{\prime}(x)(x \in D), c_{1}=c_{2}, c_{0}=c_{3}=c_{4}=0, t=1$ and $p=1$. Indeed the Kantorowitsch's hypothesis is given by

$$
c_{1} \eta \leq .5
$$

However using simple algebra, we deduce that hypothesis (7) becomes (0n $\eta$ )

$$
c_{1} \eta \leq .343145751
$$

which is a stronger hypothesis than Kantorowitsch's. However our results were primarily developed to cover the case $p \in(0,1)$ which cannot be handled with the approach in [5].

For the rest of this study we assume $A(x)=F^{\prime}(x)$ for all $x \in D$.

\section{IIII. Applications}

Example 1. Consider the real function $G$ defined on $[0, \mathrm{~s}]$ by

$$
G(x)=\frac{2}{3} x^{\frac{3}{2}}+x-3
$$

for some fixed $s>0$. 
Let $\|\cdot\|$ denote the $\max$ norm on $\mid R$. Then we get

$$
\left\|G^{\prime \prime}(x)\right\|=\max _{x \in[0, s]}\left|\frac{1}{2} x^{-\frac{1}{2}}\right|=\infty,
$$

which implies that relations (5) and (6), which are the basic assumptions for the application of the Newton-like method in [4]-[8] are not satisfied.

However it can easily be seen that $G^{\prime}(t)$ is Holder continuous on $[0, \mathrm{~s}]$ with

$$
c_{1}=1 \text { and } p=\frac{1}{2} \text {. }
$$

Therefore, under assumptions of the Theorem Newton-like iteration $\left\{x_{n}\right\}(n \geq 0)$ generated by approximation (2) converges to a solution $x^{*}$ of equation

$$
G(x)=0 .
$$

Another application for the Theorem is given by the following:

Example 2. Consider the two point boundary value problem

$$
\begin{aligned}
x^{\prime \prime}+x^{1+p} & =0, \quad p \in[0,1] \\
x(0) & =x(1)=0 .
\end{aligned}
$$

We divide the interval $[0,1]$ into $m$ subintervals and we set $h=\frac{1}{m}$. Let $\left\{v_{k}\right\} k=$ $0,1,2, \ldots, m$ be points of subdivision with

$$
p=v_{0}<v_{1}<\cdots<v_{m}=1 .
$$

A standard approximation for the second derivative is given by

$$
x_{i}^{\prime \prime}=\frac{x_{i-1}-2 x_{i}+x_{i+1}}{h^{2}}, \quad x_{i}=x\left(v_{i}\right), \quad \cdot i=1,2, \ldots, m-1 .
$$

Take $x_{0}=x_{m}=0$ and define operator $\tilde{F}: R^{m-1} \rightarrow R^{m-1}$ by

$$
\begin{aligned}
& \eta \leq \tilde{F}(x)=H(x)+h^{2} \varphi(x) \\
& H=\left[\begin{array}{cccc}
2 & -1 & & \\
-1 & 2 & -1 & 0 \\
\ddots & \ddots & \ddots \\
0 & -1 & 2 & -1 \\
& -1 & 2
\end{array}\right], \quad \varphi=\left[\begin{array}{c}
x_{1}^{1+p} \\
x_{2}^{1+p} \\
\ddots \\
x_{m-1}^{1+p}
\end{array}\right], \text { and } x=\left[\begin{array}{c}
x_{1} \\
x_{2} \\
\vdots \\
x_{m-1}
\end{array}\right]
\end{aligned}
$$

Then, we get

$$
\tilde{F}^{\prime}(x)=H+h^{2}(p+1)\left[\begin{array}{ccc}
x_{1}^{p} & & 0 \\
& x_{2}^{p} & \\
& \ddots & \\
0 & & x_{m-1}^{p}
\end{array}\right] .
$$


Newton's method cannot be applied to equation

$$
\tilde{F}(x)=0
$$

We may not be able to evaluate the second Frechet derivative since it would involve the evaluation of quantities of the form $x_{i}^{-p}$ and they may not exist.

Let $x \in R^{m-1}, H \in R^{m-1} \times R^{m-1}$ and define the norms of $x$ and $H$ by

$$
\begin{aligned}
\|x\| & =\max _{1 \leq j \leq m-1}\left|x_{j}\right| \\
\|H\| & =\max _{1 \leq j \leq m-1} \sum_{k=1}^{n-1}\left|h_{j k}\right| .
\end{aligned}
$$

For all $x, z \in R^{m-1}$ for which $\left|x_{i}\right|>0,\left|z_{i}\right|>0, i=1,2, \cdots, m-1$ we obtain, for $p=\frac{1}{2}$ say,

$$
\begin{aligned}
\left\|\tilde{F}^{\prime}(x)-\tilde{F}^{\prime}(z)\right\| & =\left\|\operatorname{diag}\left\{\left(1+\frac{1}{2}\right) h^{2}\left(x_{j}^{\frac{1}{2}}-z_{j}^{\frac{1}{2}}\right)\right\}\right\| \\
& =\frac{3}{2} h^{2} \max _{1 \leq j \leq m-1}\left|x_{j}^{\frac{1}{2}}-z_{j}^{\frac{1}{2}}\right| \leq \frac{3}{2} h^{2}\left[\max _{1 \leq j \leq m-1} \mid x_{j}-z_{j} \|^{\frac{1}{2}}\right. \\
& =\frac{3}{2} h^{2}\|x-z\|^{\frac{1}{2}} .
\end{aligned}
$$

Hence, the results in [4]-[9] cannot be applied here.

Newton's method can be seen as a system of linear equations of the form

$$
\tilde{F}^{\prime}\left(z_{n}\right)\left(z_{n}-z_{n+1}\right)=\tilde{F}\left(z_{n}\right) \quad(n \geq 0) \quad z_{0} \in R^{m-1}
$$

We choose $m=10$ which gives 9 equations. Since a solution would vanish at the endpoints and be positive in the interior a reasonable choice of initial approximation seems to be $130 x \sin \pi x$. This gives us the following vector:

$$
z_{0}=\left[\begin{array}{l}
4.01524 E+01 \\
7.63785 E+01 \\
1.05135 E+02 \\
1.23611 E+02 \\
1.29999 E+02 \\
1.23675 E+02 \\
1.05257 E+02 \\
7.65462 E+01 \\
4.03495 E+01
\end{array}\right]
$$


After 4 iterations we obtain the vector

$$
z_{4}=\left[\begin{array}{l}
3.35740 E+01 \\
6.52027 E+01 \\
9.15664 E+01 \\
1.09168 E+02 \\
1.15363 E+02 \\
1.09168 E+02 \\
9.15664 E+01 \\
6.52027 E+01 \\
3.35740 E+01
\end{array}\right]
$$

Choose $z_{4}$ as our $x_{0}$ for our Theorem. Our computations lead to the following results:

$$
\begin{aligned}
\eta & =\left\|\tilde{F}^{\prime}\left(x_{0}\right)^{-1} \tilde{F}\left(x_{0}\right)\right\|=9.15311 E-05, \quad \gamma=\left\|\tilde{F}^{\prime}\left(x_{0}\right)^{-1}\right\|=2.55882 E+01 \\
c_{1} & =c_{2}=\frac{3}{2} h^{2} \gamma=0.015 \beta=.383823, \quad c_{0}=c_{3}=c_{4}=0, \quad p=\frac{1}{2},
\end{aligned}
$$

and by inequalities (7) and (9), we obtain

$$
d=9.18 E-05>\eta \text { and } R=1.563202728 .
$$

It can easily be seen that with the above values all hypotheses of the Theorem are satisfied. Hence Newton's iteration $\left\{z_{n}\right\}(n \geq 0)$ generated by approximation (21) is well defined, remains in $U\left(x_{0}, d\right)$ and converges to a solution $x^{*}$ of equation (20) which is unique in $U\left(x_{0}, R\right)$.

\section{References}

[1] I. K. Argyros, Newton-like methods under mild differentiability conditions with error analysis, Bull. Austral. Math. Soc. 37(1987), 18-23.

[2] - - On the secant method and fixed points of nonlinear equations, Monatsheffe fru Mathematik, 106(1988), 85-94.

[3] —- A convergence theorem for Newton-like methods under generalized Chen-Yamamoto type assumptions, Appl. Math. Comp. 61(1994), 25-37.

[4] I. K. Argyros and F. Szidarovszky, The Theory and Application of Iteration Methods, C. R. C. Press, Inc. Boca Raton, Florida, 1993.

[5] L. V. Kantorowitsch and G. P. Akilov, Functional Analysis in Normed Spaces. Oxford Publ. Pergamon Press, N. Y., 1964.

[6] I. Pavaloiu, Observations concerning some approximation methods for the solutions of operator equations, Revue d'analyse Numerique et de theorie de I'approximation, 23(1994), 185-195.

[7] F. A. Potra and V. Ptak, Nondiscrete Induction and Iteration Processes. Pitman Publ., New York, 1984. 
[8] _- Sharp error bounds for Newton's process, Numer. Math. 34(1980), 63-72.

[9] F. A. Potra, Sharp error bounds for a class of Newton-like methods, Libertas Mathematica 5(1985), 71-84.

[10] F. Szidarovszky and O. Palusinski, A special matrix equation and its applications in microelectronics, Appl. Math. Comp. 64(1994), 115-119.

Cameron University, Department of Mathematics, Lawton, OK 73505, U. S. A. 